\title{
Chronic Pancreatitis and Malabsorption - Defects Involved and Physiological Consequences
}

\author{
Riddhima Banerji* \\ Department of Gastroenterology, Royal London Hospital, Barts Health NHS Trust, \\ London \\ *Corresponding Author: Riddhima Banerji, Department of Gastroenterology, Royal \\ London Hospital, Barts Health NHS Trust, London.
}

Received: July 03, 2021

Published: November 11, 2021

(C) All rights are reserved by Riddhima

Banerji.

\begin{abstract}
Intestinal malabsorption is a complex clinical condition with multiple adverse consequences. An understanding of the basic principles underlying the process of nutrient absorption is, therefore, essential. The normal assimilation and digestion of essential nutrients depends on the optimal motor, secretory and absorptive functions of the gastrointestinal system. The enteric nervous system plays an important role in this process. The physiology of absorption is complex. This is possibly because multiple functions of the GI system, both at the cellular and organic levels, must be carefully orchestrated together in order to bring about effective absorption and digestion. Therefore, defects in this intricate system would lead to the malabsorption of nutrients, with malnutrition as the result. The most common causes of malabsorption in the United Kingdom are coeliac disease, Crohn's disease and chronic pancreatitis [1]. This review article will discuss the general mechanisms leading to malabsorption and then focus on chronic pancreatitis, the defects occurring in this condition, the physiological consequences of the malabsorption which occurs as a result of these defects and the clinical features arising as a consequence.
\end{abstract}

Keywords: Intestinal Malabsorption; Chronic Pancreatitis; Pancreatic Lipase; Steatorrhoea; Malnutrition

\section{Background}

There are multiple causes of malabsorption, involving the stomach, pancreas and intestine. The mechanisms which induce nutrient malabsorption differ in each disease condition. In the stomach, absorption is strongly related to gastric emptying, the release of gastric acid and digestive enzymes and mixing of food particles in the antrum and pylorus. In autoimmune gastritis, for instance, the reduction in gastric acid, pepsin and intrinsic factor levels leads to maldigestion. The scenario is more complex in the liver, where in biliary disease, a decrease in the availability of bile acids leads to a reduction in lipid absorption. In the intestine, a multitude of diseases can cause malabsorption. For example, in Short Bowel Syndrome, there is a reduction in the area responsible for absorption as well as a decrease in intestinal enzymes. This picture is similar to that of Coeliac disease, where villous atrophy also results in a smaller absorptive area. In Crohn's disease, intestinal tissue inflammation and injury lead to deranged levels of intestinal digestive enzymes and sub-optimal functioning of digestive enzymes. In the pancreas, multiple disease states in the pancreas lead to exocrine insufficiency. These include chronic pancreatitis, excessive alcohol ingestion, metabolic disturbances such as hypercalcaemia and hyperlipidaemia, genetic and autoimmune causes. Obstruction of the pancreatic duct, from strictures, congenital abnormalities or tumours, may also give rise to inadequate production of digestive enzymes from the pancreas, possibly as a consequence of reduced fully functional pancreatic parenchyma. It is now recognized that surgical procedures can affect the absorptive functions of the digestive system. Procedures such as the Roux-en-Y gastric 
bypass, truncal vagotomy and gastrectomy change the internal milieu of the stomach, biliary system and the pancreas, resulting in the lower production of digestive enzyme levels as well as a reduction in the potency of these enzymes, leading to malabsorption of vital nutrients.

\section{Chronic pancreatitis: clinical background}

This disease affects approximately 50/100000 people globally [2] and is characterized by the process of long-term damage to the pancreatic tissue, which is not reversible. Although alcohol is commonly attributed as the chief cause, the triggering factors for a significant proportion of the population are unaccounted for, especially in women [2]. Hence, understanding the pathophysiology of malabsorption in chronic pancreatitis is particularly relevant today, as it may point the way to identifying the precipitating causes (or causes) in patients who have an idiopathic diagnosis.

\section{Physiology of the normal aborption of nutrients}

The pancreas consists of both exocrine and endocrine tissue. Whereas the endocrine tissue secrete the hormones insulin and glucagon, it is the exocrine portion of pancreatic tissue which secretes pancreatic juice, which is responsible for providing a majority of the key digestive enzymes. These enzymes are produced from the pancreatic acinar cells, most of which are secreted as inactive precursors (zymogens), in order to prevent auto-digestion. The inactive enzymes produced include trypsinogen, proteolytic enzymes, chymotrypsinogen, procarboxypeptidase, proelastase and phospholipase $\mathrm{A}$. These are activated in the duodenum and jejunum by the enzyme enterokinase, which is present in the small intestinal epithelial tissue. Enzymes which are secreted in their active form include lipase, alpha-amylase, ribonuclease and deoxyribonuclease.

Pancreatic juice enters the second part of the duodenum along with bile (which enters via the bile duct) through the Ampulla of Vater, a process which is controlled by the sphincter of Oddi. Under normal circumstances, this sphincter remains contracted between meals and relaxes only when food has been ingested and the digestion process is initiated. Following relaxation of the sphincter, the junction opens, allowing bile and pancreatic juice to enter the small intestine. When these fluids enter the small intestine, they mix with the digestive juices produced by the intestinal walls, along with chyme from the stomach. The pancreatic juice contains a high pro- portion of bicarbonate ions, which creates an alkaline (or neutral) environment within the small intestine, thereby allowing optimum conditions for the digestive enzymes to interact with their respective nutrient substrates. This is because it is essential for the gastric acid to be neutralized by an alkaline fluid (the pancreatic juice), in order to allow the intestinal enzymes to act on their substrates.

It can therefore be concluded that the pancreas plays a central role in absorption. This can further be appreciated by the functional abnormalities occurring in Chronic Pancreatitis and Cystic Fibrosis, two disease conditions where there is a loss of functioning pancreatic parenchyma and a resulting decrease in the production of digestive enzymes.

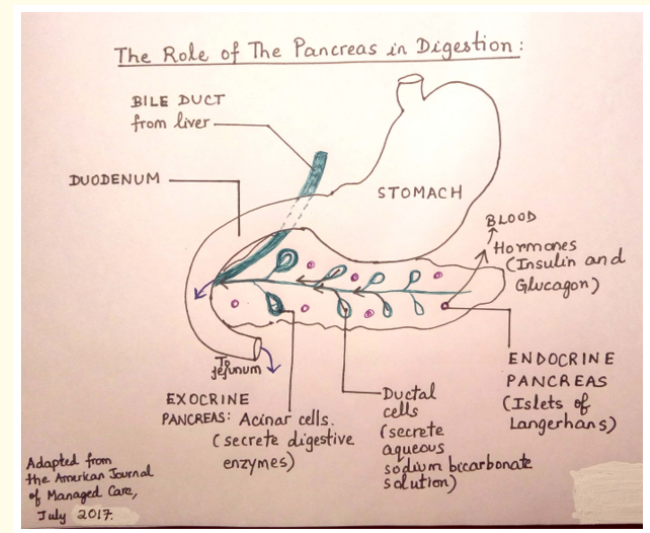

Figure 1: Adapted from the American Journal of Managed Care, Jul 2017.

\section{Case History}

A 40 year old man with a history of alcohol abuse presented to his physician with complaints of continuous epigastric pain, which radiated through to the back. He had similar episodes of recurring pain over the past year. He had lost a significant amount of weight. His general physical examination was remarkable for jaundice. The patient's stools were pale, and bulky, suggestive of steatorrhoea. Laboratory investigations revealed high levels of bilirubin, alkaline phosphatase and glucose in the blood in addition to glycosuria, confirming the presence of diabetes. CT scan of the abdomen showed an oedematous pancreas and a diagnosis of Chronic Pancreatitis was made [3]. 


\section{Discussion}

\section{Defects involved}

The key dysfunction in chronic pancreatitis is inadequate secretion of bicarbonate ions and water by the pancreatic ducts. This leads to a relatively higher protein level in the ductal pancreatic juice. These proteins, over a length of time, ultimately precipitate into the pancreatic secretory system, resulting in the evolution of protein plugs, which accumulate inside the ducts. A series of problems ensue as a result of this development. To begin with, the formation of protein plugs causes a blockage in the pancreatic drainage system. In order to accommodate the backflow of digestive fluids, the proximal ducts begin to dilate. The resulting increase in back pressure has two detrimental effects. Firstly, this causes the classical penetrating abdominal pain of this disease. A possible reason for the occurrence of pain may be an impingement on the coeliac plexus of nerves by the oedematous pancreas. Inflammation leading to hyper-responsiveness and disturbance in the functioning of the autonomic nerves surrounding the plexus may also be a cause for the severe pain of this condition. This series of events may also explain why repeated attacks of acute pancreatitis lead to the development of chronic pancreatitis, which is sometimes the case in alcohol related disease (as in the patient mentioned above).

Long-standing inflammation leads to the insidious destruction of pancreatic parenchyma and the gradual replacement of normal tissue with fibrotic tissue. This process occurs in conjunction with the ongoing impairment of acinar, islet and ductal cell function. Unfortunately, this is a relentless process, wherein the damage caused is permanent and irreversible and both pancreatic exocrine and endocrine insufficiency ensue. This inflammation may eventually, also spread to adjacent organs, such as the bile duct and the duodenum and even ascend up to the stomach and reach as far down as the transverse colon.

The pancreas has the unique capacity of having immense tissue reserve, and the ability to adapt to an onslaught of damaging events. This may be a reason why exocrine pancreatic insufficiency and underlying mal-absorption of essential nutrients may not surface as clinical signs and symptoms for a considerably long time. Therefore, it is essential for the clinician to have a high index of suspicion so as to avoid missing a diagnosis of malnutrition (arising as a consequence of malabsorption).
Interestingly, the reason behind malabsorption of nutrients in chronic alcohol consumption is yet to be ascertained. It is thought that alcohol may trigger the precipitation of proteins and initiate an inflammatory process. These proteins contain a high concentration of calcium phosphate and calcium bicarbonate, the presence of which may cause inflammation of the surrounding tissue and exacerbate tissue injury and destruction. They may also precipitate as stones. Toxic by-products of alcohol may also directly stimulate local fibroblast cells and accelerate change in the surrounding tissue morphology.

\section{Clinical consequences of chronic inflammation in the pancreas}

The obstruction of the secretory ducts and reduction of acinar cells has multifold effects. In the initial phase, there is a reduced production of alkaline juice and pancreatic enzymes. The decreased activity of digestive enzymes in the small bowel leads to reduced absorption of nutrients. As a consequence, mal-absorption and loss of weight follow. The loss of pancreatic lipase is particularly relevant, as it is responsible for $90 \%$ of fat absorption. Thus the malabsorption of fats is more pronounced than that of proteins and carbohydrates and usually occurs before the mal-digestion of other macronutrients. The next step in this process is reduced micelle formation and the precipitation of bile salts, leading to an attenuated bile salt pool. This in turn results in the formation of a vicious cycle of reduced fat digestion and impaired absorption of fat soluble micronutrients, such as vitamins A, D, E and K [4]. Fat which has failed to be thus absorbed is excreted in the form of pale, bulky stools which float on water, owing to air trapped in the undigested fat molecules. This is known as steatorrhoea (present in this patient). Clinically, steatorrhoea tends to be an early detrimentally affected. Lipase is also particularly sensitive to harm from an acidic environment. However, this process occurs usually when the pancreatic lipase production falls below $10 \%$ of the normal level. In the later phase of the disease, clinical manifestations of vitamins A deficiency (night blindness), vitamin D deficiency (rickets and osteoporosis), vitamin E deficiency (myopathy, immune system compromise) and vitamin $\mathrm{K}$ deficiency (bruising and bleeding) take place.

There are other clinical manifestations of chronic inflammation in the pancreas

These include fibrosis of the inferior end of the common bile duct, resulting in constriction and a narrowing of the duct lumen.

Citation: Riddhima Banerji. "Chronic Pancreatitis and Malabsorption - Defects Involved and Physiological Consequences". Acta Scientific Gastrointestinal Disorders 4.12 (2021): 13-16. 
Pancreatic stellate cells are primarily responsible for the regulation of the fibrosis process and the formation of prominent extracellular matrix [5]. As a consequence, there is cessation of bile flow and a spill-over of bile into the systemic circulation. This is reflected as high serum bilirubin. Cellular necrosis also occurs in the biliary system. The enzyme alkaline phosphatase is produced by the damaged cells and reflected as a high serum alkaline phosphatase level (as in this patient).

\section{Conclusion}

The pathophysiology behind malabsorption in Chronic Pancreatitis is complex. Pancreatic Exocrine Insufficiency is characterized by a severe reduction in intestinal pancreatic activity. The clinical manifestations of this condition are largely a result of impaired activity of pancreatic lipase, namely steatorrhoea, weight loss and the complications arising from malnutrition. Future work may involve a targeted approach to investigate the genetic and molecular changes in pancreatic acinar cells, which may be a possible reason for the malabsorption of nutrients in patients with Chronic Pancreatitis. Pancreatitis may involve the activation of the anit-aging gene Sirtuin 1. Sirtuin 1 is critical to prevent programmed cell death in various cell and tissues and Sirtuin 1 may be relevant to defects in chronic pancreatitis and malabsorption [6-9].

I would like to thank the editorial board of Acta Scientific Journal of Gastrointestinal disorders for their comments and suggestions.

\section{Bibliography}

1. Tidy C. "Gastrointestinal Malabsorption". The Information Standard, 28th December (2016).

2. Gupte A and Goede D. "Chronic Pancreatitis". BMJ 7th June 361 (2018): k2126.

3. Smith ME and Morton DG. "Exocrine Functions of The Pancreas". The Digestive System, (2016 reprint), second edition, Churchill Livingstone (2016): 72-84.

4. Al-Kaade S. Exocrine Pancreatic Insufficiency, emedicine.medscape.com, January 10th (2019).

5. Hackert T., et al. "The Pancreas: Causes for Malabsorption". Viszeralmedizin, June 30.3 (2014): 190-197.

6. Ian J Martins. "Anti-Aging Genes Improve Appetite Regulation and Reverse Cell Senescence and Apoptosis in Global Populations". Advances in Aging Research 5 (2016): 9-26.
7. Ian Martins. "Single Gene Inactivation with Implications to Diabetes and Multiple Organ Dysfunction Syndrome". Journal of Clinical Epigenetics 3.3 (2017): 24.

8. Ian Martins. "Nutrition Therapy Regulates Caffeine Metabolism with Relevance to NAFLD and Induction of Type 3 Diabetes". Journal of Diabetes and Metabolic Disorders 4 (2017): 019.

9. Wauters E., et al. "Sirtuin-1 regulates acinar-to-ductal metaplasia and supports cancer cell viability in pancreatic cancer". Cancer Research 73.7 (2013): 2357-2367.

10. Figure 1: Adapted from the American Journal of Managed Care, Jul (2017).

Volume 4 Issue 12 December 2021

(C) All rights are reserved by Riddhima Banerji. 